\title{
Oxigenoterapia domiciliaria
}

\author{
M. Lina Boza C. ${ }^{\text {; }}$ Hortensia Barrientos L. '; Juan Badilla S. ${ }^{1}$; \\ Francisco Prado A. ${ }^{1,2}$; M. Edith Vergara B. ${ }^{3}$
}

\section{Home oxygen therapy for children with chronic respiratory diseases}

\begin{abstract}
Twenty :ive children with oxygen deoendent chronic respiratory insufficiency were prospectively eval Jated to determine if home oxygen therapy was feasible. All potients had chronic respirotory insufficiency with arteriai blocd hemoglobin oxigen saturalion $93 \%$ or less wher breathing at ambiert air and prolonged suppiemental oxyger needs. Their disease wos secondon to severe viral diseose [n: 10 ) oystic fibrosis (n: 5 ). bronchopulmonary dysplosio (n; 3) or other causes (n: 7) [genetic diseases and mulkple malformations were excluded]. Patients were predominanty maie 22: 11: in most of them (60\%) oxygen therapy was storted belore age three years. Nine patients were dismissed from the study becouse of family problems or out of town homes. Sixteen patients went home under axygen therapy after appropriate Iraining of their parents. Hol: of them were able lo stop axygen therapy affer an average 11.9 morths from hospital dischaige. No complicotions derived from home oxygen therapy were recorded. Social, educoional or economical disadvantoges were not obsiccles lo comply with medical indicalions. The cosl of home oxygen treatmenl was approximareiy $U \$ \$ 4$ per morith plus an iritiai investrment of $U \$ \$ 46$ in necessary equipment versus $U 2887$ per manth of hospilal management. Fome oxygen therapy is feasible and free of accidents when families are properly motivated and trained. This proctice is adyantageous lor patients and lamilies in terms of emolional benefits, money soving and risk of nosocomial ineclions.
\end{abstract}

Key words: !ung disease, chronic, home care senvices, hospilalized thila, hospitalization, length of slay, oxygen therapy.t

Cada vez más pacientes sobreviven con afecciones respiratorias severas, daño pulmonar e hipoxemia crónica. Las causas más frecuentes de tratamiento prolongado con oxígeno en los niños son el nacimiento prematuro con displasia broncopulmonar como secuela, enfermedades virales graves por adenovirus u otros virus, fibrosis quística y las afecciones neuromusculares. Todas ellas producen disfunción pulmonar severa, deterioro de la relación ventilación-perfusión, hipoxemia de reposo con o sin retención de $\mathrm{CO}_{2}$ y alteración del equilibrio de ácidos y bases. Además del tratamiento específico de la enfermedad causal, estos pacientes

1. Hospital San Borja-Arriarán, Servicio de Pediatría, Unidad de Enfermedades Respiratorias. Servicio de Salud Metropolitano Central.

2. Universidad de Chile, Facultad de Medicina, Departamento de Pediatría, Campus Centro.

3. Enfermera Lniverșitaria. Hospital San Borja-Arriarán, Servicio de Pedjatría, Unidad de Enfermedades Respiratorias. necesitan suplementos de oxígeno para alcanzar saturaciones de hemoglobina iguales o mayores de $95 \%$ y prevenir los efectos de la hipoxemia crónica, como hipertensión pulmonar secundaria a vasoconstricción del lecho vascular pulmonar, corazón pulmonar y poliglobulia ${ }^{\mathrm{I}}$. El tratamiento prolongado con oxígeno en pacientes con displasia broncopulmonar disminuye la duración de las hospitalizaciones, el riesgo de infección intrahospitalaria, evita la producción de corazón pulmonar y disminuye la letalidad de la afección ${ }^{5-7}$.

El proposito de este trabajo fue determinar si en nuestro medio es posible aplicar con éxito un programa de oxigenoterapia domiciliaria, sobre la base de una experiencia de tres años en una unidad de manejo de enfermedades respiratorias infantiles.

\section{Material y Método}

En eneto de 1992 se inició el programa de oxigenoterapia domiciliaria en pacientes de la unidad de enferrmeda- 
des respiratorias del Servicio de Pediatría del Hospital San (Francisco de) Borja - (Manuel) Arriarán de Santiago, Chile. Este informe corresponde a la evaluación de los niños oxígeno dependientes admitidos al hospital entre esa fecha y diciembre de 1994. En todos los casos las saturaciones arteriales de bemoglobina eran de $93 \%$ o menores cuando respiraban aire ambiental y se requería permanentemente oxígeno por dos o más meses durante el sueño, la actividad física a la alimentación, para alcanzar saturación de oxígeno de la hemoglobina entre $95 \%$ y $97 \%$. Se excluyeron de la experiencia domiciliaria los pacientes que sufrian enfermedades genéticas, malformaciones múltiples o cuyas familias no fuesen capaces de cumplir el programa. Todos los pacientes seleccionados fueron evaluados mediante un protocolo de seguimiento que incluía aspectos epideniojogicos, clínicos y sociales.

El informe de las condiciones socioeconómicas famjliares y de factibilidad para instalar el balón de oxígeno en la casa estuvo a cargo del servicio de asistencia social. El equipamiento domicliario consistente en balones portátiles de oxígeno, flujometro, mantometro, humedecedor y nariceras. fue comprado por la familia u ottas instituciones, mientras que el balón fijo, con la carga de oxigeno necesaria y su reposicion en el domicilio, fueron de cargo del hospital.

Los pacientes fueron enviados a su domicilio sólo si estaban estables clínica y radiologicamente. sin modjficaciones en su tratamiento durante al inenos 15 días, con necesidades de oxígeno por naricera igual o menor a 21 - min, sin retencion importante de $\mathrm{CO}_{2}$ (igual o menor a $50 \mathrm{mmHg}$ ) y con indicaciones que pudiesen ser cumplidas sir dificultad en la casa. Antes del alta de cada paciente se dio información detallada a la familia sobre la enfermedad de aquél e instrucciones de enfermería para su manejo y tratamiento, todo lo cual fue incluido en un snanual escrito. Los conocínientos y el entreaamiento requeridos fueron entregados a los fatniliares durante la hospilalización del nifo, durante la cual su madre o cuidadora pudo acompanarto diariamente.

En el curso de la hospitalización los pacientes recibieron broncodilatadores beta 2 agonistas en los periodos de reagudización de los síntomas obstructivos; esteroides orales en curas de una semana ( $2 \mathrm{mg} \cdot \mathbf{k g}$ • día) cuando no hubo respuesta satisfactoria con beta 2 agonistas; antibióticos si las manifestaciones clínicas y de laboratorio asi to indicaban, además del tratamiento específico de la enfermedad de base e insnunización antivirus influenza con las cepas recomendadas anualmente para este efecto (OPS/ OMS). La alimentación se prescribio a base de formulas con 150 a $170 \mathrm{kcal} \cdot \mathrm{kg}$ - día con alta proporción de lípidos (44\%), baja en hidratos de carbono ( $44 \%$ ) y el resto en proceĺnas. Indicaciones similares se dejaron al alia del paciente.

En los diferentes pacientes se realizaron controles semanales, quincenales o mensuales después del alta, dependiendo de las necesidades individuales, pam lo cual se usó un local separado del policlínico de la especialidad con objeto de evitar las infecciones cruzadas con olros pacientes ambulatorios. El seguimiento incluyó evaluación del estado nutricional, medicion de saturación de oxigeno de la hemoglobina con oxímetro de pulso, examen clínico, los exămenes de laboratorio que se estimasen necesarios, radiografías de tórax y electrocardiograma.
Se encuestó a la madre o persona a cargo del niōo sobre su nivel de instruccion; cumplimiento de las instrucciones dadas al alta; sentimientos de angustia, rabia, miedo o tristeza; necesidad de consultar a otro médico u otro tipo de expento en medicina no alopática (homeópatas, curanderos, etc.); apoyo emocional u econónico de amigos u otros miembros de la familia, repercusión sobre la pareja (discusiones, separación, frustración, sentimientos de culpa); calidad de las destrezas adquiridas durante la hospitalización del paciente y comprensión de la enfermedad.

Desde el inicio del programa cumplían el criterio de oxígeno dependencia 25 pacientes, de los cuales siete provenian de provincias, adonde fueron devueltos con indicaciones precisas para su manejo. Dos ninos fueron referidos a otras instinuciones por incapacidad de la familia para participar en el programa. Los restantes 16 pacientes ingresaron al programa de ox́geno terapia domiciliaria. En el conjunto de los 25 pacientes oxígeno dependientes el diagnóstico más frecuente fue enfermedad viral confirmada con inmunofluorescencia indirecta ([FI) viral positiva para adenovirus en tres casos y sospechada clínicamente y en el laboratorio en siete, totalizando 10 pacientes $(40 \%)$. Cinco pacientes padecian fibrosis quistica (20\%), tres displasia broncopulmonar $(12 \%)$. El resto estaba constituido por un caso cada uno de inmunodeficiencia, enfermedad de Mikity Wilson, artritis reumatoide, síndrome de Arnold Chiari, atrofia espinal progresiva, enfermedad de los cilios inmoviles y gangliosidosis. En las radiografíns de tórax se encontraron una 0 más de las siguientes alteraciones: condensación y atelectasia en 14 pacientes ( $56 \%$ ): biperinsuflación en 10 (43\%); engrosamiento peribronquial en 9 (37\%) y bronquiectasia en 8 (31\%). La edad de inicio de la oxígeno dependencia de los veinticinco pacientes iniciales estaba entre dos meses y 16 ános: en 60\% de los casos el ingreso ocurrió antes de los 3 años (tabla) y el comienzo de las síntomas respiratorios se produjo entre el nacjmiento y la edad de 3 años, a los 6,8 meses en promedio. Diecisétis pacientes eran varones (proporción varón:mujer $=2: 1$ ) El estado nutricional previo a la oxigenoterapia era normal en siete niños (43\%), cualro se consideraron en riesgo de desnutrición (25\%) y el resto sufría desnutrición de prinier o segundo grado.

El alta se programó en forma escalonada, es decir, se dio inicialmente por 24 o 48 horas, al cabo de las cuales e] paciente reingres6, fue cvaluado y luego reenviado a casa por periodos cada vez más largos, hasta que la familia se sintió segurá.

\section{Resultados}

El tiempo de mantención con oxigenoterapia de los pacientes que pudieron abstenerse final-

Tabla

Distribución por edad

\begin{tabular}{lrr}
\hline Edad & n & $\%$ \\
\hline $2 \mathrm{~m}-3 \mathrm{a}$ & 15 & 60 \\
$3 \mathrm{a}-6 \mathrm{a}$ & 4 & 16 \\
$6 \mathrm{a}-9 \mathrm{a}$ & 1 & 4 \\
$9 \mathrm{a}-12 \mathrm{a}$ & 4 & 16 \\
$+12 \mathrm{a}$ & $\mathrm{1}$ & 4 \\
\hline
\end{tabular}


mente de ella fue de 11,9 meses en promedio y al término de la evaluación había ocho de ellos sin oxígeno (50\%) y cuatro que sólo empleatan oxígeno en la noche -durante el sueño- y cuando hacían ejercicio. De los diez pacientes en los cuales el trastorno se atribuy 6 a una enfermedad viral, cuatro $(40 \%)$ pudieron suspender la terapia con oxígeno en un promedio de 9 meses. Los demás siguen en tratamiento. La fibrosis quística es la afección que ha requerido períodos más largos de tratamiento, alcanzando 22,6 meses como promedio al término de esta evaluación.

El número promedio de hospitalizaciones previas era, en 14 de los 16 pacientes que ingresaron al programa, 5,5, y la duracion de ellas fue 41,6 (márgenes 25 a 369) días. La causa del ingreso era reagudización de sus síntomas respiratorios y deterioro de la condición clínica con hipoxemia severa. Dos pacientes nunca habían sido dados de alta desde que nacieron, a causa de displasia broncopulmonar y necesidad permanente de oxígeno suplementario. Después de ingreso al plan sólo tres pacientes se rehospitalizaron por períodos de 3,3 días en promedio, fundamentalmente por reagudización de los síntomas de obstrucción de la via aérea baja. Sólo falleció un paciente que sufría de daño pulmonar por adenovirus, quien a los 11 meses de edad babía sufrido otra infección por virus sincicial respiratorio, desarrollando insuficiencia respiratoria progresiva, por lo que recibió ventilación mecánica. Este niño murió seis meses después del comienzo de la oxigenoterapia ambulatoria. El estado nutricional de los cinco pacientes desnutridos no se modificó sustancialmente, pese al buen aporte calórico y correspondieron a casos de displasia broncopulmonar, inmunodeficiencia, gangliosidosis y atrofia espinal progresiva.

La encuesta social al grupo familiar reveló que la madre tom 6 la responsabilidad del cuidado del niño en 11 casos (66\%), la abuela en cuatro $(22 \%)$ y otro familiar en el restante. El nivel de instrucción de la persona a cargo era: en siete pacientes, básica $(43,7 \%)$, media en ocho $(50 \%)$ y en un caso analfabetismo sin instrucción, a pesar de lo cual no se registraron diferencias en el cumplimiento de las indicaciones médicas y de enfermeria.

En siete $(43,7 \%)$ casos se registraron alteraciones emocionales del grupo familiar con sen- timientos iniciales de negación de la enfermedad, angustia, miedo e inseguridad en la capacidad de enfrentar el problema una vez que el niño fuera dado de alta, lo que fue superado enviando a la madre o persona a cargo a apoyo psicológico y reforzando las indicaciones médicas y de enfermería permanentemente. Despues del regreso del enfermo a la casa, los familiares de cinco de ellos $(31,3 \%)$ sintieron necesidad de consultar otra opinión, incluyendo curanderos e iriólogos, y en 10 casos se alteró seriamente la vida familiar $(62,5 \%)$, con separación de la pareja en uno.

No se reportaron accidentes en el manejo de los equipos en domicilio, a pesar que 10 pacientes viven en casas de construcción ligera en regulares condiciones de mantención y aceptables condiciones higiénicas.

\section{Comentario}

Hay numerosos informes sobre los beneficios del manejo domiciliario con oxígeno y otras medidas, en pacientes con insuficiencia respiratoria crónica secundaria a displasia broncopulmonar ${ }^{1,3-7}$, sin embargo no existen datos publicados sobre la evolución clínica y el tiempo que duran las demandas suplementarias de oxígeno en otras afecciones, fundamentalmente el daño pulmonar secundario a virus, como es el caso en nuestra serie, en que este diagnóstico fue el más frecuente.

Enviar pacientes a su casa con oxígeno significa una reducción muy importante de los costos de hospitalización. El coste estimado de un día cama es de aproximadamente $\$ 38500$ (U\$ $\$ 6$ ), lo que significa un $\$ 1155000$ (U\$ 2887 ) mensuales. En contraste, el gasto que implica el suministro domiciliario de oxígeno con un consumo promedio de dos cilindros de $10 \mathrm{~m}^{3}$ mensuales, a un coste de $\$ 20060\left(\$ 1003 \mathrm{~m}^{3}\right)$, más $\$ 1734$ (\$867 cada uno) por concepto de transporte de los balones, el coste total mensual es de \$21 794 (U\$ 54). La adquisición de flujómetro, manómetro, humidificador, nariceras y cilindro de traslado implican un gasto inicial de $\$ 218548$ (U\$ 546) en total, suma que ha sido aportada en la mayor parte de nuestros casos por las municipalidades respectivas. Estos implementos quedan, al término del tratamiento, disponibles para su uso por otros niños, pues la 
vida útil de los cilindros y carros de transporte es de 10 años y del resto -excluyendo la naricera- es 5 afios.

El tratamiento de estos pacientes en su casa produce inicialmente alteraciones emocionales y de la vida intrafamiliars. Para resolver estas dificultades, al menos en parte, se requiere un tiempo de adaptación y apoyo psicológico a la familia. Sin embargo, además de disminuir los costes de atencion, el manejo domiciliario evita los peligros de la hospitalización prolongada, incluyendo las infecciones intrahospitalarias y las alteraciones psicológicas inherentes a la separación del niño de su familia.

Las diferentes condiciones socioeconómicas y de instrucción de los padres no han representado inconvenientes en el entrenamiento de la familia y el manejo de los enfermos por parte de sus parientes, aun cuando la persona a cargo sea analfabeta, como en uno de nuestros pacientes que aún sigue en tratamiento.

Tanto en los casos con una enfernedad de base viral confirmada o sospechada, como en niños con displasia broncopulmonar, puede mirarse el futuro con optimismo, pues si se cumplen las recomendaciones de control y tratamiento, el pronostico de la función pulmonar mejora progresivamente hasta hacer posible la suspensión del suministro adicional de oxígeno, lo que guarda estrecha relación con el crecjmiento y desarrollo de pulnón sano, que compensa paulatinamente el daño adquirido ${ }^{1}$. Las dificultades en la recuperación nutricional que muestran algunos pacientes probablemente tiene cierta rejacion con el cambios en el gasto energético y las alteraciones de la mecánica pulmonar a que los somete su enfermedad ${ }^{9.10}$, pero también con dificultades materiales para ofrecer aportes nutricionales apropiados en el hogar y dificultades del propio paciente para alimentarse.

Así pues, el tratamiento con oxígeno en el domicilio es seguro si la familia está bien entrenada, disminuye el riesgo de reingreso al hospital, evitando las infecciones intrahospitalarias y reduce el coste de atención de salud. Desafortunadamente, debido a la variable velocidad de recuperación de los distintos pacientes, es imposible predecir con precisión el tiempo de dependencia al oxígeno en cada caso y los eventuales reingresos al hospital, pero se pueden hacer aproximaciones que estimulan el optimismo de la familia sobre el futuro de su niño. Estos resultađos muestran que es posible hacer tratamiento con oxígeno en el hogar con ventajas médicas y sociales, a pesar de sus limitaciones provenientes del pequeño tamaño de la muestra y la falta de un grupo control.

\section{Resumen}

Se describen los resultados de una experiencia con oxigenoterapja domiciliaria en niños con afecciones respiratorias crónicas dependientes de aportes suplementarios de oxígeno, con el prop6sito de mostrar su factibilidad y ventajas sobre el manejo intrahospitalario. Los pacientes seleccionados provenían de un total de 25 niños que ingresaron a la unidad de enfermedades respiratorias de un hospital docente de Santiago por insuficiencia respiratoria crónica, con saturaciones de oxígeno de $93 \%$ o menores de la hemoglobina arterial, respirando en aire ambiental, secundaria a daño pulmonar por infecciones virales severas ( $n: 10)$, displasia broncopulmonar ( $n$ : 3), fibrosis quística (n: 5) y otras afecciones ( $\mathrm{n}: 7$ ) del aparato respiratorio. Todos requerían tratamiento con oxígeno suplementario por largo tiempo. La edad de comienzo de la dependencia a oxígeno, en $60 \%$ de los casos, fue previa a los 3 años. Predominaban los varones $(2: 1)$. Se escogieron 16 niños para tratamiento en sus domicilios, previo entrenamiento de las personas a cargo, con indicaciones precisas de manejo, que se cumplieron sin mayores inconvenientes. Los nueve restantes fueron enviados a la ciudad de su procedencia en provincias (n: 7) o a instituciones, por incapacidad de la familia para cumplir el tratamiento en el hogar. El tiempo promedio de permanencia en el programa de los pacientes en que finalmente fue posible suspender la oxígenoterapia $(50 \%)$ fue 11,9 meses. El tratamiento domiciliario produce beneficios emocionales a los pacientes, disminución del riesgo de infecciones intrahospitalarias y ahorro en los gastos de atención de salud. El coste del tratamiento en la casa es de $\$ 21770$ (U\$ 54) mensuales, con una inversion inicial de $\$ 218548$ (U\$ 546) que se compara favorablemente con el coste de mantencion de un niño en el hospital, que es de $\$ 1155000$ (U\$ 2 887) mensual. 
(Palabras clave: enfermedades pulmonares, cuidados domiciliarios, nin̄os hospitalizados, duración de las hospitalizaciones, oxígeno terapia.)

\section{Agradecimientos}

A las Asistentes Sociales del Hospital San Borju-Arriarán. Sra. Rosa Inostroza y Srta. Rosa Alarcón, por su generosa colaboración.

\section{Referencias}

1. Abman $S H$, Bancalari E: Bronchopulmonary dysplasia conference. Ped, Pulmonol 1987; 3: 185-196.

2. Goodman $G$, Perkin RM. Anas NG: Pulmonary hypertension in infants with bronchopulmonary dysplasia. J Pediatr 1988: 112: 67-72.

3. National Heart, Lung and Blood Instirte. Workshop on bronchopulmonary dysplasia. I Pediatr 1979: 85: $9208,95: 829-834$.
4. MC Aleese KA. Knapp MA, Rhodes TT: Financial and emotional cost of bronchopulmonary dysplasia. Clin Pediatr (Phila) 1993; 32: 393-400.

5. Srahiman M: Clinical description of bronchopulmonary dysplasia. J Pediatr 1979; 95: 829-834.

6. Abman SH, Wolfe RR, Accurso PS: Pulmonary vascular response to oxygen in infants with severe bronchopulmonary dysplasia, Pediatrics 1985; 75: 80-84

7. Campbell $A N$, Zarfin $Y$, Groenveld $M$ : Low flow oxygen therapy in infants. Arch Dis Child 1983; 58: 785-789.

8. Rush $M G$, Hasinski TA: Current therapy of bronchopulmonary dysplasia. Clin Perinatol 1992; 19: 563-590.

9. Me Aleese KA, Knapp MA. Rhodes TT: Fibancial and emotional cost of bronchopulmonary dysplasia. Clin Pediatr (Phila) 1993; 32: 393-400.

10. Kurzner SI, Garg $M$ : Growth failure in bronchopulmonary dysplasia: Elevated metabolic rates and pulmonary mechanics. J Pediatr 1988; 112: 73-80.

11. Singer L, Martin RJ. Hawkins SW: Oxygen desaturation complicates feeding in infants with bronchopulmonary dysplasia after discharge. Pediatrics 1992; 90 : $380-384$. 